\title{
Expression of vascular endothelial growth factor (VEGF) and its receptors in thyroid carcinomas of follicular origin: a potential autocrine loop
}

Joaquim Miguel Vieira ${ }^{1}$, Susana C Rosa Santos ${ }^{1}$, Carla Espadinha ${ }^{1}$, Isabel Correia ${ }^{1}$, Tibor Vag $^{1}$, Cristina Casalou ${ }^{1}$, Branca Maria Cavaco ${ }^{1}$, Ana Luísa Catarino ${ }^{2}$, Sérgio Dias ${ }^{1}$ and Valeriano Leite ${ }^{1,3}$

${ }^{1}$ Centro de Investigação de Patobiologia Molecular (CIPM), ${ }^{2}$ Departmento de Patologia Morfológica, Instituto Português de Oncologia Francisco Gentil - Centro Regional de Oncologia de Lisboa (CROL), S.A, Rua Professor Lima Basto, 1099-O23 Lisboa, Portugal and ${ }^{3}$ Faculdade de Ciências Médicas da Universidade Nova de Lisboa, Lisboa, Portugal

(Correspondence should be addressed to V Leite, CIPM, Instituto Português de Oncologia Francisco Gentil; Email: vleite@ipolisboa.min-saude.pt)

\begin{abstract}
Objective: The aim of this study was to clarify the role of vascular endothelial growth factor (VEGF) and VEGF receptor (VEGFR) pathways in thyroid tumourigenesis.

Methods: We examined VEGF, VEGFR-1 and VEGFR-2 expression on 34 papillary thyroid carcinomas (PTCs), 18 follicular thyroid carcinomas (FTCs), eight poorly differentiated thyroid carcinomas (PDTCs) and on a thyroid tumour-derived cell line (NPA'87) by immunohistochemistry, reverse transcriptase PCR, immunofluorescence and Western blotting.

Results: We have demonstrated that VEGF expression was significantly $(P<0.05)$ more prevalent in PTCs $(79 \%)$ than in FTCs $(50 \%)$ or PDTCs (37\%). Similarly, $76 \%$ of PTCs, $83 \%$ of FTCs and $25 \%$ of PDTCs expressed VEGFR-1, whereas $68 \%$ of PTCs, 56\% of FTCs and 37\% of PDTCs expressed VEGFR-2. Coexpression of VEGF and its receptors was observed in 50\% of PTCs, $39 \%$ of FTCs and $12 \%$ of PDTCs, raising the possibility that VEGF may signal in an autocrine loop in these neoplasias, as observed previously for other types of cancer. In agreement with the idea that autocrine VEGF signalling plays an important role in thyroid carcinogenesis, the blockade of either VEGF or its receptors with neutralizing antibodies significantly reduced cell viability and increased apoptosis levels of the VEGFR-positive thyroid tumour cell line NPA'87.

Conclusions: Our results highlight a previously undefined VEGF autocrine action in thyroid carcinomas which could play a crucial role in tumour cell survival and could represent a useful therapeutic target for thyroid tumours.
\end{abstract}

European Journal of Endocrinology 153 701-709

\section{Introduction}

Vascular endothelial growth factor (VEGF) plays a key role in tumour angiogenesis, an essential step in tumour growth. VEGF expression is upregulated in the majority of human tumours, where it stimulates proliferation, migration and survival of endothelial cells by binding to two high-affinity tyrosine kinase receptors, VEGFR-1/Flt-1 and VEGFR-2/KDR. VEGF receptors (VEGFRs) are mainly expressed by endothelial cells, although they have also been detected in other cell types, including macrophages, monocytes (1) and platelets (2). More recently, it was shown that both VEGF and its receptors are expressed in haematological (3), pancreatic (4) and prostatic (5) tumour cells, where they may serve as autocrine stimulators of proliferation, migration and survival.
Most thyroid tumours arise from follicular thyroid cells. They comprise a wide spectrum of neoplastic phenotypes, including benign adenomas, well-differentiated papillary and follicular thyroid carcinomas (PTCs and FTCs respectively) and aggressive poorly differentiated and undifferentiated thyroid carcinomas (PDTCs and UTCs respectively). A number of studies have examined the expression of VEGF and its receptors in human thyroid carcinomas with reference to angiogenesis $(6-10)$. VEGF expression is upregulated in thyroid neoplasia, and correlates with the size, spread and recurrence of tumours. For example, VEGF and VEGFR1 expression correlate with PTC size in children and adults (9), thyroid tumours that have metastasized have higher levels of VEGF than nonmetastatic disease, correlating with a worse prognosis (8), and lymph-node metastases of thyroid carcinomas showed increased VEGF expression compared with primary tumour (7). 
Whereas VEGF expression is well documented, much less is known about VEGFR expression in thyroid carcinomas, and some studies obtained contradictory results $(6,8,9)$. For instance, some reports describe VEGFR-1 expression as restricted to endothelial cells of normal thyroid tissue, PTCs and FTCs $(6,8)$. However, others (9) reported VEGFR-1 expression also in PTC cells, raising the possibility of an autocrine mechanism for VEGF on thyroid follicular cells. Expression of VEGFR-2 has been detected in thyroid follicular cells but only at the mRNA level (7).

During the process of malignant transformation, several events have been shown to regulate the expression of VEGF and its receptors, such as hypoxia, p53 deficiency and Ras-activated signal transduction pathway (11). Activating point mutations of B-Raf, particularly the Val-600 $\rightarrow$ Glu (V600E) mutation in the activation segment of the kinase domain, have been found in $29-83 \%$ of conventional PTCs but not in FTCs (12-16). To date, the correlation between VEGF, VEGFR expression and B-Raf point mutations in thyroid carcinomas has not been analysed.

In this study, we examined the expression pattern and biological function of VEGF and its receptors in vivo and in vitro to clarify the role of this angiogenic pathway in thyroid tumourigenesis. We demonstrate for the first time the presence of a functional autocrine VEGF/VEGFR pathway that could represent a useful target for therapeutic intervention in thyroid carcinomas.

\section{Materials and methods}

\section{Thyroid tissue collection and cell culture}

Human thyroid tissues, obtained from fresh surgical samples, were fixed in formalin and embedded in paraffin within $3 \mathrm{~h}$ of collection. For the current study, 60 specimens were studied, including 34 PTCs (20 classic variants, 11 follicular variants, 2 tall-cell variants and one cribiform variant), 18 FTCs (15 minimally invasive and three extensively invasive) and eight PDTCs. The extent of PTCs was classified according to the system of DeGroot et al. (17) and the metastasis/age/completeness of resection/invasion size score (MACIS; (18)). The system of DeGroot et al. (17) categorizes the patients with PTC by clinical class: I, with intrathyroidal disease; II, with cervical adenopathies; III, with extrathyroidal invasion and IV, with distant metastasis. The prognostic score defined as MACIS was calculated as: MACIS $=3.1$ (if aged $\leq 39$ years) or $0.08 \times$ age (if aged $\geqq 40$ years), $+0.3 \times$ tumour size (in $\mathrm{cm}$ ), +1 (if incompletely resected),+1 (if locally invasive) and +3 (if distant metastasis is present).

NPA'87 cells (kindly provided by Dr Danilo V. Canlapan, Los Angeles School of Medicine, Los Angeles, CA, USA) were cultured at $37^{\circ} \mathrm{C}$ in RPMI 1640 (Life Technologies, Gaithersburg, MD, USA) supplemented with 10\% foetal bovine serum, sodium pyruvate, L-glutamine and penicillin/streptomycin, in a humidified incubator with $5 \% \mathrm{CO}_{2}$.

\section{Immunohistochemical analysis}

For immunohistochemistry, $2 \mu \mathrm{m}$ paraffin-embedded thyroid carcinoma sections were deparaffinized and treated with $0.6 \% \mathrm{H}_{2} \mathrm{O}_{2}$ in methanol to inhibit endogenous peroxidase. Microwave antigen retrieval was used to enhance immunoreactivity. Tissues were blocked with 10\% normal goat serum (X907; Dako, Golstrup, Denmark) at room temperature for $10 \mathrm{~min}$, before blocking the endogenous avidin and biotin (SP2001; Vector Laboratories, Burlingame, CA, USA). The antibodies used were polyclonal rabbit antihuman VEGF serum (Santa Cruz Biotechnology, Santa Cruz, CA, USA), monoclonal mouse anti-human VEGFR-1 (clone FB5; kindly provided by ImClone Systems, New York, NY, USA) and monoclonal mouse anti-human VEGFR-2 (clone 6.64; kindly provided by ImClone Systems). Primary antibodies were incubated at $4{ }^{\circ} \mathrm{C}$ overnight. This was followed by sequential incubations with biotin-conjugated secondary antibody, streptavidin/peroxidase and 3,3'-diaminobenzidine (K5001; Dako) for visualization. Sections were counterstained with Mayer's haematoxylin. As negative controls primary antibodies were omitted.

\section{Assessment of microvessel density (MVD)}

Blood vessel content in 29 specimens (14 FTCs and 15 PTCs) was determined by immunostaining with a monoclonal rabbit anti-human von Willebrand factor (vWF) antibody (Dako). A modified version of the Weidner method (19) was used for MVD assessment. Briefly, by scanning the tumour sections at low power $(40 \times)$, the amount of intratumoural vessels was graded from 1 to 4 . Then, three areas with the highest vessel density were selected in the tumour, and individual vessels were counted at high power $(200 \times)$. MVD values represent the average of the assessed 'hotspots'.

\section{Reverse transcriptase (RT)-PCR}

Total RNA was reverse-transcribed using Superscript II (Life Technologies). Single-stranded cDNA was amplified using Taq polymerase (Life Technologies) as recommended by the manufacturer. VEGF, VEGFR-1 and VEGFR-2 PCR amplification was done with specific oligonucleotide pairs, described previously (3).

\section{Immunofluorescence analysis}

$\mathrm{NPA}^{\prime} 87$ cells were grown on glass coverslips until $70 \%$ confluent, washed with PBS, and then fixed in $4 \%(\mathrm{v} / \mathrm{v})$ formaldehyde/PBS for $5 \mathrm{~min}$ at $4^{\circ} \mathrm{C}$ and washed in PBS. After permeabilization with $0.1 \%(\mathrm{v} / \mathrm{v})$ Triton X-100 
plus 5\% (v/v) normal serum, cells were incubated overnight at $4^{\circ} \mathrm{C}$ with primary antibodies (polyclonal rabbit anti-human VEGFR-1 or VEGFR-2, both from Santa Cruz Biotechnology). Cells were washed and incubated with secondary antibody (Alexa fluor 594; Molecular Probes, Eugene, OR, USA) for $1 \mathrm{~h}$ at room temperature. Samples were mounted in Vectashield (Vector Laboratories) and analysed using fluorescence microscopy (Axioplan Microscope; Zeiss, Cologne, Germany). For negative controls primary or secondary antibodies were omitted.

\section{Protein extraction and Western blotting}

Nonidet P-40 lysates were obtained by resuspending the NPA'87 cells in the following buffer: $1 \%$ Nonidet P-40, $50 \mathrm{mM}$ Tris, pH 7.5, 10\% glycerol and protease/phosphatase inhibitors. Equal protein amounts were separated by SDS/PAGE and transferred to nitrocellulose membranes. Blots were incubated with polyclonal antibodies raised against VEGFR-1, VEGFR-2 or phosphotyrosine (Santa Cruz Biotechnology).

\section{Cell-viability assay}

$\mathrm{NPA}^{\prime} 87$ cells were cultured in the presence or absence of the neutralizing monoclonal antibody (mAb) for VEGF (clone 4.6 .1 at $100 \mathrm{ng} / \mathrm{ml}$; kindly provided by Genentech, South San Francisco, CA, USA), VEGFR-1 (clone 6.12 at $1 \mu \mathrm{g} / \mathrm{ml}$; kindly provided by ImClone Systems) or VEGFR-2 (clone IMC1C11 at $1 \mu \mathrm{g} / \mathrm{ml}$; kindly provided by ImClone Systems). After 24, 48 and $72 \mathrm{~h}$, viable and dead cells were counted in triplicate, based on trypan blue exclusion. Each experimental condition was performed in duplicate, and experiments were repeated three times.

\section{Apoptosis studies}

$\mathrm{NPA}^{\prime} 87$ cells were treated with vehicle or neutralizing $\mathrm{mAb}$ to VEGF (clone 4.6.1), VEGFR-1 (clone 6.12) or VEGFR-2 (clone IMC1C11) for $24 \mathrm{~h}$. Cells were washed once in the incubation buffer (10 mM Hepes, $\mathrm{pH} 7.4,10 \mathrm{mM} \mathrm{NaCl}$ and $5 \mathrm{mM} \mathrm{CaCl}_{2}$ ) and resuspended in incubation buffer supplemented with $0.5 \mathrm{mg} / \mathrm{ml}$ propidium iodide (Sigma-Aldrich, St Louis, MO, USA) and annexin V-FITC (Boehringer Mannheim, Mannheim, Germany) at room temperature for $30 \mathrm{~min}$. The percentage of early apoptotic cells (annexin V-FITC-positive, propidium iodide-negative) was determined by flow cytometry (FACScan; Becton-Dickinson, San Jose, CA, USA). These experiments were repeated three times.

\section{Cell-cycle analysis}

$\mathrm{NPA}^{\prime} 87$ cells were treated as described for apoptosis studies, washed in cold PBS containing $5 \mathrm{mM}$ EDTA, then incubated in the above buffer supplemented with $0.2 \mathrm{mg} / \mathrm{ml}$ RNase A, at room temperature, for $30 \mathrm{~min}$. Propidium iodide solution $(5 \mu \mathrm{g} / \mathrm{ml})$ was added to the samples and cell cycle was analysed by flow cytometry. DNA histograms were obtained by using Multicycle software (Phoenix Flow Systems, San Diego, CA, USA).

\section{Mutation screening of B-Raf}

We performed mutation analysis in exon 15 of $B$-Raf in 20 conventional PTCs. The complete coding sequences of exon 15 of $B$-Raf were amplified using exonic primers and directly sequenced on both strands using the ABI PRISM $^{\circledR}$ Big Dye ${ }^{\mathrm{TM}}$ Terminator Cycle Sequencing Ready Reaction Kit and analysed by the ABI PRISM ${ }^{\mathrm{TM}}$ 310 Genetic Analyser (Applied Biosystems, Foster City, CA, USA). The primer $\left(5^{\prime}-3^{\prime}\right)$ sequences were as follows: B-Raf forward primer, GCC AAG TCA ATC ATC CAC AG; B-Raf reverse primer, CAT CTG ACT GAA AGC TGT ATG GA.

\section{Statistical analysis}

All graphs with error bars indicate mean \pm s.E.M. The relationship between VEGF immunoreactivity and MVD was assessed using the Spearman correlation test. VEGF and VEGFR immunostaining was compared
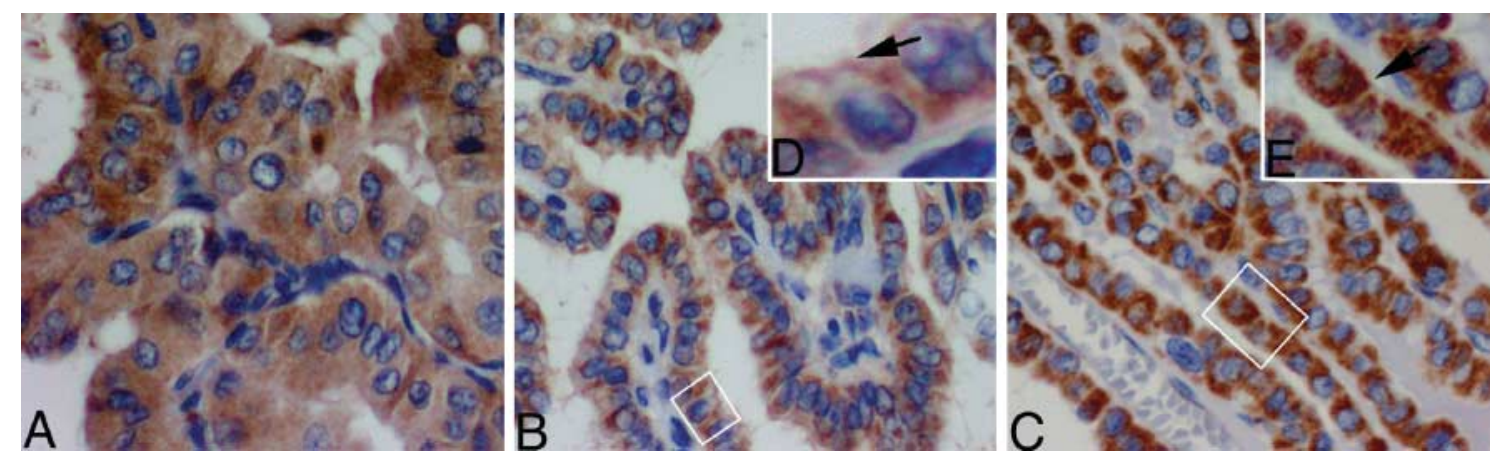

Figure 1 Immunohistochemical detection of VEGF (A), VEGFR-1 (B, D) and VEGFR-2 (C, E) in cytoplasm and on the surface (D, E) of thyroid carcinoma cells. Arrows indicate VEGFRs staining in cell membrane. Panels are a series from three different thyroid carcinomas. Original magnifications, $A-C, \times 400 ; D$ and $E, \times 600$. 
between different tumour histotypes by two-tailed Fisher's exact test. The correlation of VEGF and VEGFR expression with clinical/pathological features of the patients or the presence of $B-R a f$ point mutations was performed using unpaired t-test, two-tailed Fisher's exact test or $\chi^{2}$ test, as appropriate. Differences between experimental and control groups in cell viability, apoptosis and cycle assays were evaluated by oneway analysis of variance (ANOVA), followed by Tukey's post-hoc test. Statistical analysis was performed using GraphPad Prism statistical software (San Diego, CA, USA). Differences were considered statistically different at $P<0.05$.

\section{Results}

\section{Expression of VEGF and VEGFRs in human thyroid carcinomas}

VEGF immunohistochemical staining was detected in $79 \%(27 / 34)$ of PTCs $(P=0.03$ versus FTC; $P=0.02$ versus PDTC), $50 \%(9 / 18)$ of FTCs $(P>0.05$ versus PDTC) and $37 \%(3 / 8)$ of PDTCs. The VEGF immunoreactivity was always confined to the cytoplasm of epithelial cells (Fig. 1), but an occasional inflammatory
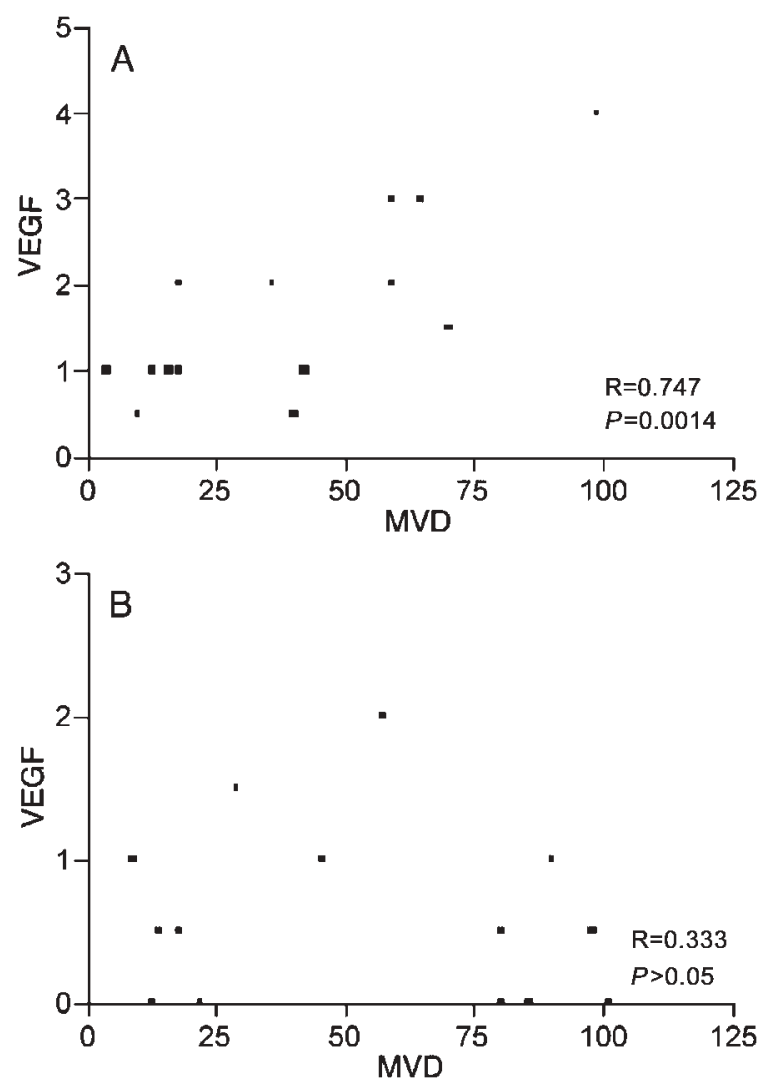

Figure 2 Correlation between MVD and VEGF expression in PTCs (A) and FTCs (B). Spearman correlation test was used to determine $r$ and $P$ values. cell staining was also observed (data not shown). VEGF levels in FTCs and PTCs were compared with MVD. The correlation between VEGF expression and MVD was statistically significant in PTCs $(r=0.747$; $P=0.0014)$ but not in FTCs $(r=0.333 ; P>0.05$; Fig. 2).

Immunohistochemical staining with antibodies specific for VEGFR-1 or VEGFR-2 revealed that, besides staining the endothelial lining of blood vessels, subsets of carcinoma cells also expressed the receptors (Fig. 1). VEGFR-1 immunoreactivity was detected in $76 \%$ $(26 / 34)$ of PTCs $(P>0.05$ versus FTC; $P=0.006$ versus PDTC), 83\% (15/18) of FTCs $(P=0.004$ versus PDTC) and $25 \%(2 / 8)$ of PDTCs, whereas VEGFR-2 was detected in $68 \%(23 / 34)$ of PTCs $(P>0.05$ versus FTC or PDTC), 56\% (10/18) of FTCs $(P>0.05$ versus PDTC) and 37\% (3/8) of PDTCs. In general, diffuse cytoplasmatic and membrane VEGFRs immunoreactivity was observed in thyroid tumour cells (Fig. 1). Coexpression of VEGF and its receptors was observed in 50\% (17/34) of PTCs $(P>0.05$ versus FTC or PDTC), 39\% (7/18) of FTCs $(P>0.05$ versus PDTC) and 12\% (1/8) of PDTCs.

\section{Relationship between clinicopathological data and the expression of VEGF and its receptors in human thyroid carcinomas}

FTCs expressing VEGF, VEGFR-1, VEGFR-2 or both VEGF and its receptors (VEGF + VEGFRs) were not significantly larger or more invasive than those lacking its expression (Table 1). In PTC cases (Table 2), we observed that $95 \%(19 / 20)$ of the tumours with a classic pattern were positive for VEGFR-1, whereas only $45 \%(5 / 11)$ of the follicular variants were positive $(P=0.007)$. Additionally, we observed that $65 \%$ $(13 / 20)$ of conventional PTCs were positive for both VEGF and its receptors, whereas only 18\% (2/11) of the follicular variants expressed both VEGF and VEGFRs $(P=0.03)$. Interestingly, $100 \%(5 / 5)$ of the tumours positive for both VEGF and VEGFRs had metastasized to neck lymph nodes (class II tumours), whereas only $32 \%(6 / 19)$ of intrathyroidal tumours (class I tumours) were positive $(P=0.04)$.

\section{Mutation screening of B-Raf}

Since VEGF and its receptors were mainly expressed by conventional PTCs, we next investigated whether there was a correlation with the presence of $B-R$ af mutations, a common event in this kind of tumours. We detected the B-Raf ${ }^{\mathrm{V} 600 \mathrm{E}}$ mutation in 50\% (10/20) of conventional PTCs, but there was no significant correlation between the presence of the B-Raf ${ }^{\mathrm{V} 600 \mathrm{E}}$ mutation and the expression of VEGF and its receptors (Table 3). 
Table 1 Clinical and pathological features and VEGF/VEGFR expression in FTCs.

\begin{tabular}{|c|c|c|c|c|c|c|c|c|c|c|c|c|}
\hline & \multicolumn{3}{|c|}{ VEGF } & \multicolumn{3}{|c|}{ VEGFR-1 } & \multicolumn{3}{|c|}{ VEGFR-2 } & \multicolumn{3}{|c|}{ VEGF + VEGFRs } \\
\hline & \multicolumn{2}{|c|}{ Immunostaining } & \multirow[b]{2}{*}{$P$ value } & \multicolumn{2}{|c|}{ Immunostaining } & \multirow[b]{2}{*}{$P$ value } & \multicolumn{2}{|c|}{ Immunostaining } & \multirow[b]{2}{*}{$P$ value } & \multicolumn{2}{|c|}{ Immunostaining } & \multirow[b]{2}{*}{$P$ value } \\
\hline & Negative & Positive & & Negative & Positive & & Negative & Positive & & Negative & Positive & \\
\hline Tumour size (cm \pm S.D.) & $3.4 \pm 1.9$ & $4.9 \pm 1.3$ & NS & $4.3 \pm 1.3$ & $3.9 \pm 1.9$ & NS & $3.4 \pm 1.1$ & $4.8 \pm 2.0$ & NS & $3.6 \pm 1.8$ & $5.1 \pm 1.1$ & NS \\
\hline Invasiveness & & & NS & & & NS & & & NS & & & NS \\
\hline Minimally invasive $(n=15)$ & $8(53 \%)$ & $7(47 \%)$ & & $3(20 \%)$ & $12(80 \%)$ & & $8(53 \%)$ & $7(47 \%)$ & & $10(67 \%)$ & $5(33 \%)$ & \\
\hline Extensively invasive $(n=3)$ & $1(33 \%)$ & $2(67 \%)$ & & $0(0 \%)$ & $3(100 \%)$ & & $0(0 \%)$ & $3(100 \%)$ & & $1(33 \%)$ & $2(67 \%)$ & \\
\hline Distant metastasis $(n=3)$ & $1(33 \%)$ & $2(67 \%)$ & NS & $0(0 \%)$ & $3(100 \%)$ & NS & $0(0 \%)$ & $3(100 \%)$ & NS & $1(33 \%)$ & $2(67 \%)$ & NS \\
\hline \multicolumn{13}{|l|}{ Clinical status } \\
\hline Persistent $(n=3)$ & $1(33 \%)$ & $2(67 \%)$ & NS & $0(0 \%)$ & $3(100 \%)$ & NS & $0(0 \%)$ & $3(100 \%)$ & NS & $1(33 \%)$ & $2(67 \%)$ & NS \\
\hline Remission $(n=15)$ & $8(53 \%)$ & $7(47 \%)$ & & $3(20 \%)$ & $12(80 \%)$ & & $8(53 \%)$ & $7(47 \%)$ & & $10(67 \%)$ & $5(33 \%)$ & \\
\hline
\end{tabular}

NS, not significant.

Table 2 Clinical and pathological features and VEGF and VEGFR expression in PTCs.

\begin{tabular}{|c|c|c|c|c|c|c|c|c|c|c|c|c|}
\hline & \multicolumn{3}{|c|}{ VEGF } & \multicolumn{3}{|c|}{ VEGFR-1 } & \multicolumn{3}{|c|}{ VEGFR-2 } & \multicolumn{3}{|c|}{ VEGF + VEGFRs } \\
\hline & \multicolumn{2}{|c|}{ Immunostaining } & \multirow[b]{2}{*}{$P$ value } & \multicolumn{2}{|c|}{ Immunostaining } & \multirow[b]{2}{*}{$P$ value } & \multicolumn{2}{|c|}{ Immunostaining } & \multirow[b]{2}{*}{$P$ value } & \multicolumn{2}{|c|}{ Immunostaining } & \multirow[b]{2}{*}{$P$ value } \\
\hline & Negative & Positive & & Negative & Positive & & Negative & Positive & & Negative & Positive & \\
\hline Tumour size ( $\mathrm{cm} \pm$ S.D.) & $3.7 \pm 1.8$ & $3.8 \pm 2.7$ & NS & $3.5 \pm 1.4$ & $3.9 \pm 2.8$ & NS & $3.8 \pm 1.4$ & $3.8 \pm 2.9$ & NS & $4.1 \pm 1.6$ & $3.7 \pm 1.8$ & NS \\
\hline MACIS (mean \pm S.D.) & $5.6 \pm 1.8$ & $5.4 \pm 1.6$ & NS & $4.9 \pm 0.9$ & $5.6 \pm 1.6$ & NS & $5.2 \pm 1.0$ & $5.7 \pm 1.9$ & NS & $5.6 \pm 1.7$ & $5.8 \pm 1.9$ & NS \\
\hline Class & & & NS & & & NS & & & NS & & & 0.04 \\
\hline I $(n=19)$ & $5(26 \%)$ & $14(74 \%)$ & & $7(37 \%)$ & $12(63 \%)$ & & $8(42 \%)$ & $11(58 \%)$ & & $13(68 \%)$ & $6(32 \%)$ & \\
\hline$\|(n=5)$ & $0(0 \%)$ & $5(100 \%)$ & & $0(0 \%)$ & $5(100 \%)$ & & $0(0 \%)$ & $5(100 \%)$ & & $0(0 \%)$ & $5(100 \%)$ & \\
\hline III $(n=8)$ & $1(12 \%)$ & $7(88 \%)$ & & $1(12 \%)$ & $7(88 \%)$ & & $3(37 \%)$ & $5(63 \%)$ & & $3(37 \%)$ & $5(63 \%)$ & \\
\hline IV $(n=2)$ & $1(50 \%)$ & $1(50 \%)$ & & $0(0 \%)$ & $2(100 \%)$ & & $0(0 \%)$ & $2(100 \%)$ & & $1(50 \%)$ & $1(50 \%)$ & \\
\hline Predominant pattern & & & NS & & & 0.007 & & & NS & & & 0.03 \\
\hline Classic $(n=20)$ & $3(15 \%)$ & 17 (85\%) & & $1(5 \%)$ & $19(95 \%)$ & & $5(25 \%)$ & $15(75 \%)$ & & 7 (35\%) & $13(65 \%)$ & \\
\hline Follicular $(n=11)$ & $3(27 \%)$ & 8 (73\%) & & $6(55 \%)$ & $5(45 \%)$ & & $5(45 \%)$ & $6(55 \%)$ & & $9(82 \%)$ & $2(18 \%)$ & \\
\hline Others $(n=3)$ & $1(33 \%)$ & $2(67 \%)$ & & $1(33 \%)$ & $2(67 \%)$ & & $1(33 \%)$ & $2(67 \%)$ & & $1(33 \%)$ & $2(67 \%)$ & \\
\hline Clinical status $^{\mathrm{a}}$ & & & NS & & & NS & & & NS & & & NS \\
\hline Persistent $(n=6)$ & $1(17 \%)$ & 5 (83\%) & & $1(17 \%)$ & $5(83 \%)$ & & $2(33 \%)$ & $4(67 \%)$ & & $2(33 \%)$ & $4(67 \%)$ & \\
\hline Remission $(n=27)$ & $6(22 \%)$ & $21(78 \%)$ & & $7(26 \%)$ & 20 (74\%) & & $9(33 \%)$ & $18(67 \%)$ & & $15(56 \%)$ & $12(44 \%)$ & \\
\hline
\end{tabular}


Table 3 Correlation of $B$-Raf mutations with VEGF and VEGFR expression in conventional PTCs.

\begin{tabular}{|c|c|c|c|c|c|c|c|c|c|c|c|c|}
\hline & \multicolumn{3}{|c|}{ VEGF } & \multicolumn{3}{|c|}{ VEGFR-1 } & \multicolumn{3}{|c|}{ VEGFR-2 } & \multicolumn{3}{|c|}{ VEGF + VEGFRs } \\
\hline & \multicolumn{2}{|c|}{ Immunostaining } & \multirow[b]{2}{*}{$P$ value } & \multicolumn{2}{|c|}{ Immunostaining } & \multirow[b]{2}{*}{$P$ value } & \multicolumn{2}{|c|}{ Immunostaining } & \multirow[b]{2}{*}{$P$ value } & \multicolumn{2}{|c|}{ Immunostaining } & \multirow[b]{2}{*}{$P$ value } \\
\hline & Negative & Positive & & Negative & Positive & & Negative & Positive & & Negative & Positive & \\
\hline$B-R a f^{V 600 E}$ & & & NS & & & NS & & & NS & & & NS \\
\hline Positive $(n=10)$ & $2(20 \%)$ & $8(80 \%)$ & & $0(0 \%)$ & $10(100 \%)$ & & 3 (30\%) & 7 (70\%) & & $3(30 \%)$ & $7(70 \%)$ & \\
\hline Negative $(n=10)$ & $1(10 \%)$ & $9(90 \%)$ & & $1(10 \%)$ & $9(90 \%)$ & & $2(20 \%)$ & $8(80 \%)$ & & $4(40 \%)$ & $6(60 \%)$ & \\
\hline
\end{tabular}

NS, not significant.

\section{A VEGF/VEGFR autocrine loop in NPA'87 cells is crucial for survival}

The coexpression of VEGF and VEGFRs on thyroid carcinomas of follicular origin, particularly in conventional PTCs, suggests the presence of a VEGF autocrine loop. In order to test this hypothesis, we used specific neutralizing antibodies to block the VEGF/VEGFR pathway in a human papillary thyroid carcinoma-derived cell line, $\mathrm{NPA}^{\prime} 87$. These cells express the three VEGF isoforms (VEGF-121, VEGF-165 and VEGF-189) as well as both VEGFRs; VEGFR-1/Flt-1 and VEGFR2/KDR (Fig. 3A). The VEGFR expression was further characterized by immunofluorescence (Fig. 3B). VEGFR-1 staining was evident both in the cytoplasm and on the cell membrane of $\mathrm{NPA}^{\prime} 87$ cells, while VEGFR-2 staining, also evident in the cytoplasm, showed a more prominent perinuclear pattern (Fig. 3B). However, only VEGFR-2 seems to be functionally active on $\mathrm{NPA}^{\prime} 87$ cells, as demonstrated by Western blotting (Fig. 3C). After stimulation with VEGF, no VEGFR-1 phosphorylation was observed (Fig. 3C). Noteworthy, in the absence of exogenous VEGF, baseline VEGFR-2 phosphorylation was observed in NPA'87 cells (Fig. 3C), further suggesting the existence of a functional autocrine loop in these cells.

Next, we investigated the potential role of the VEGF/VEGFR autocrine interaction in cell survival and cycle progression. $\mathrm{NPA}^{\prime} 87$ cells were grown in serum-free conditions and treated with neutralizing antibodies specific for VEGF or its receptors. We observed that blocking either VEGF (VEGF mAb; $100 \mathrm{ng} / \mathrm{ml}$ ) or VEGFR-2 (VEGFR-2 mAb; $1 \mu \mathrm{g} / \mathrm{ml}$ ) significantly $(P<0.05)$ reduced cell viability (live/dead ratio) by $60 \%$ (Fig. 4A), when compared with control cells (treated with vehicle), after $24 \mathrm{~h}$ of treatment. The blockade of VEGFR-1 (VEGFR-1 mAb; $1 \mu \mathrm{g} / \mathrm{ml}$ ) also decreased cell viability by $40 \%$ compared with untreated cells $(P<0.05)$. Subsequently, we evaluated cell death and cell-cycle progression in $\mathrm{NPA}^{\prime} 87$ cells treated with blocking antibodies. Using annexin VFITC- and propidium iodide-binding assay, we recorded a 2.5-fold increase in the apoptotic cell population (annexin V-FITC-positive, propidium iodide-negative) after treatment with VEGF-, VEGFR-1- or VEGFR-2neutralizing antibodies $(P<0.05$; Fig. 4B), compared with untreated cells. Conversely, cell-cycle analysis revealed a modest arrest of cells in $\mathrm{G}_{0}+\mathrm{G}_{1}$ phase, increasing approximately $17-21 \%$ compared with controls cells, and a decrease of cells in $\mathrm{G}_{2}$ phase by $26-$ $40 \%$ (Fig. 4C), independent of the treatment used. These results suggest that the VEGF/VEGFR system plays a crucial role in thyroid tumour cell survival.

\section{Discussion}

VEGF, an important stimulator of angiogenesis, has been implicated in proliferation and spread of different malignant tumours. In thyroid carcinomas of follicular origin, the role of VEGF is not fully understood. Yet, VEGF overexpression in thyroid tumours appears to be related to some clinical/pathological data of the patients $(6-10)$. VEGF overexpression correlated with PTC size in adults and children (9), and higher VEGF expression correlated with tumour metastatic behaviour and recurrence (8). In the present report, VEGF staining was found to be significantly more frequent in PTCs than in FTCs or PDTCs. Our results are in concordance with other reports (10), which also showed downregulation of VEGF in PDTCs. This finding suggests that VEGF plays an important role in the early stages of thyroid tumourigenesis, where it is overexpressed, but during progression to more undifferentiated stages, like PDTCs, other growth factors may be more important. The present study also revealed a highly significant relationship between the expression of VEGF and blood-vessel content in PTCs. This finding was contrary to MVD in FTCs, where we could not show a correlation with VEGF levels, and it is in agreement with a recent report (20). These results indicate that VEGF sustains tumour angiogenesis in PTCs, where in FTCs other angiogenic factors may be involved.

VEGF signals via two high-affinity tyrosine kinase receptors, VEGFR-1/Flt-1 and VEGFR-2/KDR, expressed mostly in endothelial cells. Recently, VEGFR expression has been reported in various types of tumour, such as leukaemia (3), pancreatic carcinoma (4) and prostatic carcinoma (5), suggesting a potential autocrine/paracrine VEGF action in these tumours. In thyroid carcinomas, VEGFR expression has been reported mainly in 
(A)

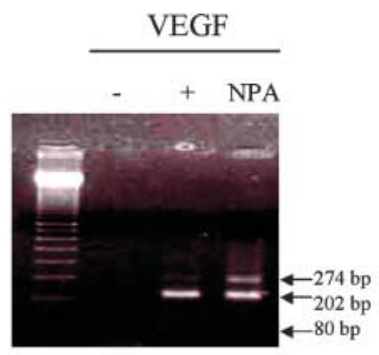

(B)

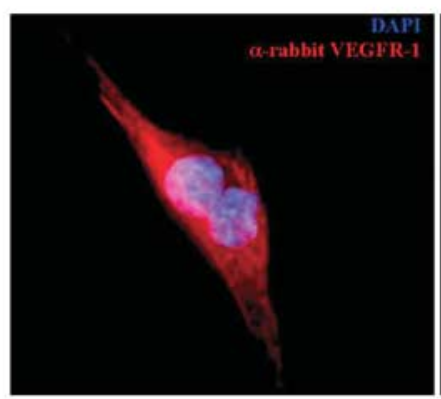

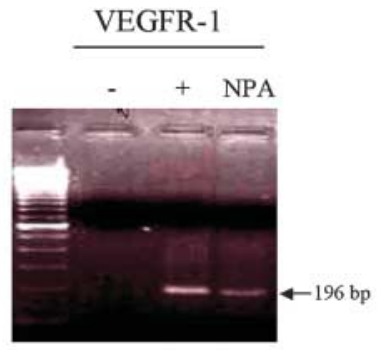

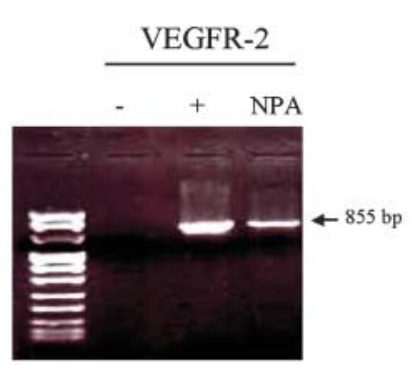

(C)

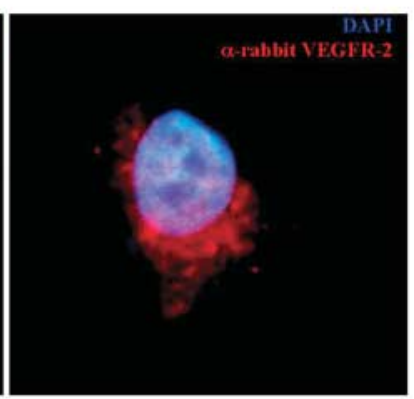

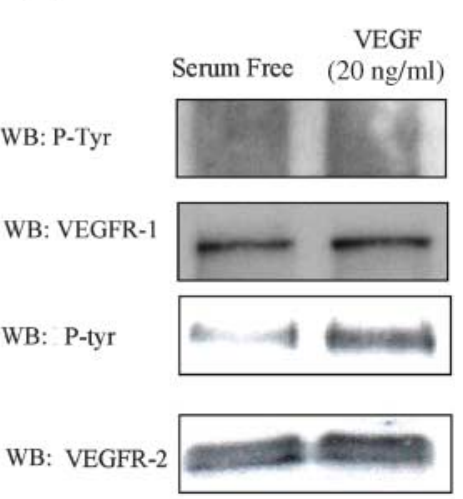

Figure 3 VEGF, VEGFR-1 and VEGFR-2 expression by NPA'87 cells. (A) RT-PCR analysis for VEGF, VEGFR-1 and VEGFR-2 in NPA'87. As a negative control (-), PCR was performed without template, whereas HL60 cDNA (VEGF) and human umbilical-vein endothelial cell cDNA (VEGFRs) were used as positive controls (+). For VEGF, the indicated bands 80, 202 and 274 bp correspond to VEGF-121, VEGF-165 and VEGF-189, respectively. For VEGFRs, the $196 \mathrm{bp}$ band corresponds to VEGFR-1, whereas the $855 \mathrm{bp}$ band corresponds to VEGFR-2. (B) Immunofluorescence analysis of NPA'87 cells in serum-free medium. These cells were stained for VEGFR-1 and VEGFR-2 (phycoerythrin) and DNA (nucleus; DAPI, 4',6-diamidino-2-phenylindole). Note the membrane and cytoplasmatic (prominent perinuclear pattern for VEGFR-2) expression of VEGFRs. This result was seen in most (95\%) cells in each experiment, and was repeated three times. Negative controls included primary antibody alone and secondary antibody alone (data not shown; original magnification, $\times 630$ ). (C) Western blot (WB) analysis of total protein extracts from NPA' 87 cells in the presence or absence (serum-free conditions) of VEGF, stained against VEGFR-1, VEGFR-2 and phosphotyrosine (p-Tyr). Note the increased phosphorylation of VEGFR-2 after VEGF stimulation $(20 \mathrm{ng} / \mathrm{ml})$ for $10 \mathrm{~min}$.

endothelial cells $(6,8)$. However, one study (9) indicated that, besides endothelial cells, VEGFR-1 might also be expressed by PTC cells. In the present study we confirm that both VEGFRs are expressed by thyroid epitheliumderived tumour cells, in addition to the endothelial cells of tumour blood vessels. This is the first demonstration of VEGFR-2 protein expression in thyroid carcinomas. Moreover, we show that coexpression of VEGF, VEGFR1 and VEGFR-2 was significantly more prevalent in classical variants than in follicular variants of PTCs, being associated with lymph-node dissemination.

We hypothesized that the presence of specific VEGFRs on thyroid carcinoma cells would allow such cells to respond to VEGF in either an autocrine or paracrine manner. To confirm this, we used a thyroid tumourderived cell line, $\mathrm{NPA}^{\prime} 87$, which expresses both VEGF and VEGFRs, as confirmed by RT-PCR, immunofluorescence and Western blotting. We investigated whether cellular processes, such as survival and cell-cycle progression were affected by inhibition of the VEGF pathway. Blockade of VEGF or VEGFRs by neutralizing
mAbs resulted in a decreased cell viability, which was more pronounced with VEGFR-2 $\mathrm{mAb}$ than with VEGFR-1 mAb. This suggests that the biological action of VEGF is mainly mediated through VEGFR-2. Blockade of VEGF/VEGFR system also induced apoptosis, but had a minor effect on cell-cycle progression. Hence, this study demonstrates a functional VEGF autocrine loop that contributes to tumour-cell survival, and suggests that VEGF- and VEGFR-specific inhibitors could be a promising approach that should be explored for treatment of thyroid tumours. Several reports have been published in support of this idea $(21-23)$. For instance, Vatalanib (PTK787/ZK222584), a specific blocker of VEGFRs (tyrosine kinase activity), was shown to inhibit the growth of thyroid carcinomas in an animal study by blocking angiogenesis (21), whereas in colorectal cancer, treatment with Bevacizumab, a VEGF-neutralizing antibody, suggested a real clinical importance (22). ZD6474, an inhibitor of VEGFR2/KDR tyrosine kinase activity, has also been shown to efficiently block oncogenic RET kinases, arresting 
(A)

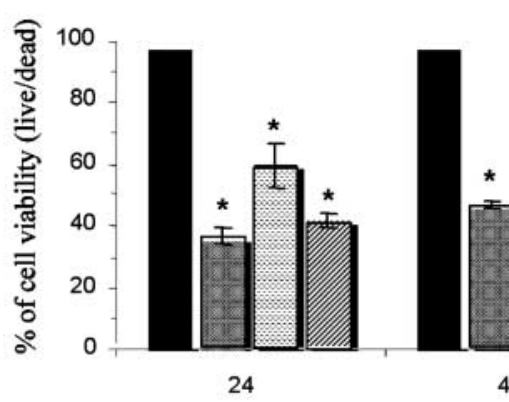

(B)

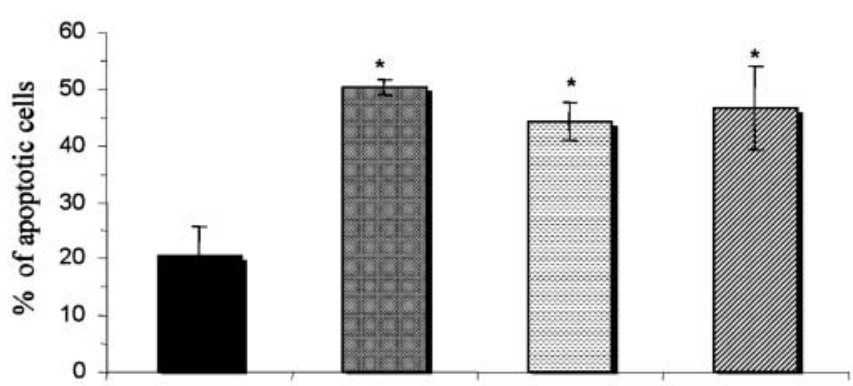

NPA

NPA + VEGF mAb

NPA + VEGFR-1 mAb

NPA + VEGFR-2 mAb

(C)

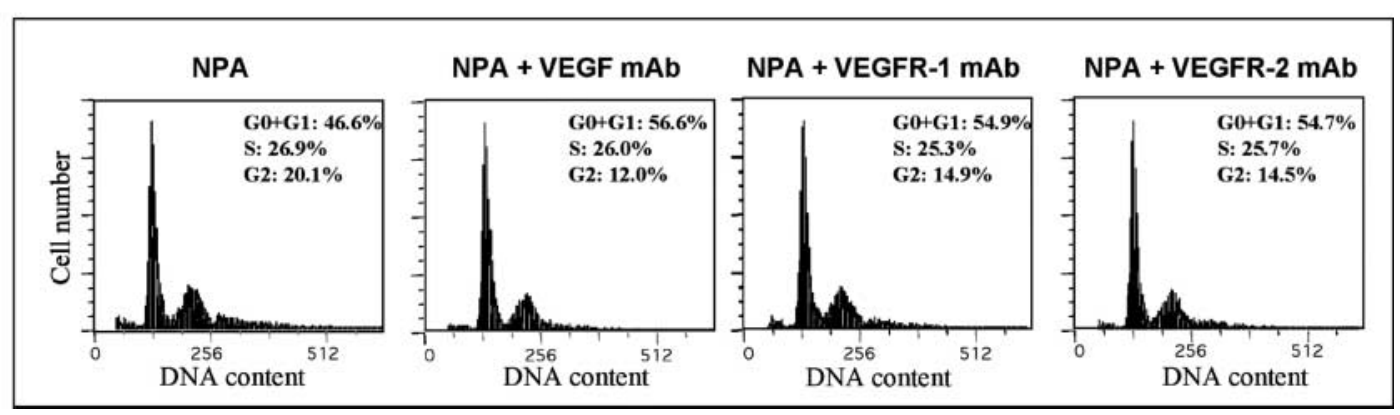

Figure 4 Blocking the VEGF/VEGFR autocrine loop exerts an effect on NPA'87 cell viability, apoptosis and cell-cycle progression. Graphs with error bars indicate means \pm S.E.M. (A) NPA' 87 cells were left untreated (in serum-free conditions) or treated with VEGF, VEGFR-1 or VEGFR-2 mAbs for 24, 48 and $72 \mathrm{~h}$. Live and dead cells (determined by Trypan Blue staining) were counted using a haemocytometer. Results show the ratio of live/dead cells in the different conditions, normalized against the untreated group. Treatment of NPA'87 cells with VEGF, VEGFR-1 or VEGFR-2 mAbs decreases cell viability ( ${ }^{\star} P<0.05$, at 24,48 and $72 \mathrm{~h}$ ), with VEGF and VEGFR$2 \mathrm{mAbs}$ having a more significant effect than VEGFR-1 mAb ( ${ }^{\star} P<0.05$, at 24 and $\left.48 \mathrm{~h}\right)$. The results shown are representative of three separate experiments. (B) Untreated (control) or treated $(24 \mathrm{~h}) \mathrm{NPA}^{\prime} 87$ cells with neutralizing mAbs against VEGF and its receptors, were stained with annexin V-FITC and propidium iodide, and analysed by flow cytometry (FACScan). Results show the percentage of early apoptotic cells (annexin V-FITC-positive, propidium iodide-negative) in the different conditions. As shown, blockade of either VEGF or its receptors induces apoptosis in NPA'87 cells $\left({ }^{\star} P<0.05\right)$. These results are representative of three independent experiments. (C) NPA'87 cells treated as before were stained with propidium iodide and analysed by flow cytometry. Results show the percentage of cells in each cell-cycle phase $\left(G_{0}+G_{1}, S\right.$ and $G_{2}$ phases).

RET/PTC3-induced tumour growth in nude mice, in addition to tumoural angiogenesis blockade (23).

Finally, we speculated about the mechanisms underlying the expression of VEGF and VEGFRs in thyroid carcinoma cells. Several events have been shown to regulate the expression of VEGF, such as hypoxia, p53 deficiency and Ras-activated signal transduction pathway (11). In fact, stimulation of the classical Ras/Raf/mitogenactivated protein kinase/extracellularsignalregulated kinase (ERK) kinase (MEK)/ERK1/2 pathway, by expression of oncogenic Ras, inducible Raf or constitutively active MEK, results in an enhanced VEGF promoter activity (24). B-Raf mutations, particularly the V600E mutation, are specifically present in classical PTCs $(12-16)$. Taking these data into consideration, we hypothesized that mutations in B-Raf could be correlated with VEGF and VEGFR expression in classical PTCs. We performed mutational analysis and detected B-Raf ${ }^{\mathrm{V} 600 \mathrm{E}}$ mutations in $50 \%$ of classical PTC, a percentage that is in agreement with previous reports (12-16). However, we did not find a correlation between the presence of $B-R a f^{\mathrm{V} 600 \mathrm{E}}$ mutation and the 
expression of VEGF or VEGFRs in PTCs. These results suggest that other mechanisms are responsible for VEGF and VEGFR overexpression in thyroid carcinomas.

In summary, coexpression of VEGF and its receptors in thyroid carcinomas, particularly in conventional PTCs, demonstrates the existence of a VEGF autocrine signalling pathway, which could contribute to tumour cell survival. Given its potential at blocking cell viability and the existence of clinically approved agents to block it, the VEGF/VEGFR pathway could represent a useful target for therapeutic intervention in thyroid carcinomas.

\section{Acknowledgements}

We gratefully acknowledge Dr Christiana Ruhrberg for critically reading the manuscript and Ms Teresa Pereira and Ms Marta Mesquita for their technical assistance in the immunohistochemical analysis. $\mathrm{T} \mathrm{V}$ is a student of the Gulbenkian PhD Programme in Biomedicine. This work was supported by Núcleo Regional do Sul Liga Portuguesa Contra o Cancro (Terry Fox), Portugal.

\section{References}

1 Sawano A, Iwai S, Sakurai Y, Ito M, Shitara K, Nakahata T \& Shibuya M. Flt1, vascular endothelial growth factor receptor 1 , is a novel cell surface marker for the lineage of monocyte-macrophages in humans. Blood 200197 785-791.

2 Selheim F, Holmsen H \& Vassbotn F. Identification of functional VEGF receptors on human platelets. FEBS Letters 2002512 107-110.

3 Dias S, Hattori K, Zhu Z, Heissig B, Choy M, Lane W, Wu Y, Chadburn A, Hyjek E, Gill M, Hicklin DJ, Witte L, Moore MA \& Rafii S. Autocrine stimulation of VEGFR-2 activates human leukemic cell growth and migration. Journal of Clinical Investigation $2000106511-521$.

4 von Marschall Z, Cramer T, Höcker M, Burde R, Plath T, Schirner M, Heidenreich R, Breier G, Riecken EO, Wiedenmann B \& Rosewicz S. De novo expression of vascular endothelial growth factor in human pancreatic cancer: evidence for an autocrine mitogenic loop. Gastroenterology $20001191358-1372$.

5 Jackson M, Roberts J, Heckford S, Ricciardelli C, Stahl J, Horsfall D \& Tilley W. A potential autocrine role for vascular endothelial growth factor in prostate cancer. Cancer Research $200262854-859$.

6 Viglietto G, Maglione D, Rambaldi M, Cerutti J, Romano A, Trapasso F, Fedele M, Ippolito P, Chiappetta G, Botti G, Fusco A \& Persico MG. Upregulation of vascular endothelial growth factor (VEGF) and downregulation of placenta growth factor (PlGF) associated with malignancy in human thyroid tumours and cell lines. Oncogene 199511 1569-1579.

7 Bunone G, Vigneri P, Mariani L, Butó S, Collini P, Pilotti S, Pierotti MA \& Bongarzone I. Expression of angiogenesis stimulators and inhibitors in human thyroid tumours and correlation with clinical pathological features. American Journal of Pathology $19991551967-1976$.

8 Klein M, Picard E, Vignaud JM, Marie B, Bresler L, Toussaint B, Weryha G, Duprez A \& Leclere J. Vascular endothelial growth factor gene and protein: strong expression in thyroiditis and thyroid carcinoma. Endocrinology $199916141-49$.
9 Fenton C, Patel A, Dinauer C, Robie D, Tuttle M \& Francis G. The expression of vascular endothelial growth and the type 1 vascular endothelial growth factor receptor correlate with the size of papillary thyroid carcinoma in children and young adults. Thyroid $200010349-357$.

10 Huang SM, Lee JC, Wu TJ \& Chow NH. Clinical relevance of vascular endothelial growth factor for thyroid neoplasms. World Journal of Surgery $200125302-306$.

11 Arbiser J. Molecular regulation of angiogenesis and tumorigenesis by signal transduction pathways: evidence of predictable and reproducible patterns of synergy in diverse neoplasms. Seminars in Cancer Biology $20041481-91$.

12 Soares P, Trovisco V, Rocha A, Lima J, Castro P, Preto A, Maximo V, Botelho T, Seruca R \& Sobrinho-Simones M. BRAF mutations and RET/PTC rearrangements are alternative events in the ethiopathogenesis of PTC. Oncogene 200322 4578-4580.

13 Fukushima T, Suzuki S, Mashiko M, Ohtake T, Endo Y, Takebayashi Y, Sekikawa K, Hagiwara K \& Takenoshita S. $B R A F$ mutations in papillary carcinomas of the thyroid. Oncogene $2003226455-6457$.

14 Namba H, Nakashima M, Hayashi T, Hayashida N, Maeda S, Rogounovitch TI, Ohtsuru A, Saenko VA, Kanematsu T \& Yamashita S. Clinical implications of hot spot BRAF mutation, V599E, in papillary thyroid cancers. Journal of Clinical Endocrinology and Metabolism $2003 \mathbf{8 8} 4393-4397$.

15 Kim KH, Kang DW, Kim SH, Seong IO \& Kang DY. Mutations of the BRAF gene in papillary thyroid carcinoma in a Korean population. Yonsei Medical Journal 200445 818-821.

16 Xing M. BRAF mutation in thyroid cancer. Endocrine-related Cancer 200512 245-262.

17 DeGroot L, Kaplan E, McCormick M \& Straus F. Natural history, treatment, and course of papillary thyroid carcinoma. Journal of Clinical Endocrinology and Metabolism 199071 414-424.

18 Hay I, Bergstralh E, Goellner J, Ebersold J \& Grant C. Predicting outcome in papillary thyroid carcinoma: development of a reliable prognostic scoring system in a cohort of 1779 patients surgically treated at one institution during 1940 through 1989. Surgery $19931141050-1057$.

19 Weidner N. Intratumoral microvessel density as a prognostic factor in cancer. American Journal of Pathology 1995 147 9-19.

20 Tanaka K, Sonoo H, Kurebayashi J, Nomura T, Ohkubo S, Yamamoto Y \& Yamamoto S. Inhibition of infiltration and angiogenesis by thrombospondin1 in papillary thyroid carcinoma. Clinical Cancer Research 20028 1125-1131.

21 Schoenberger J, Grimm D, Kossmehl P, Infanger M, Kurth E \& Eilles C. Effects of PTK787/ZK222584, a tyrosine kinase inhibitor, on the growth of a poorly differentiated thyroid carcinoma: an animal study. Endocrinology $20041451031-1038$.

22 Hurwitz H. Integrating the anti-VEGF-A humanized monoclonal antibody Bevacizumab with chemotherapy in advanced colorectal cancer. Clinical Colorectal Cancer 20044 S62-S68.

23 Carlomagno F, Vitagliano D, Guida T, Ciardiello F, Tortora G, Vecchio G, Ryan AJ, Fontanini G, Fusco A \& Santoro M. ZD6474, an orally available inhibitor of KDR tyrosine kinase activity, efficiently blocks oncogenic RET kinase. Cancer Research $2002627284-7290$.

24 Kranenburg O, Gebbink M \& Voest E. Stimulation of angiogenesis by Ras proteins. Biochimica et Biophysica Acta Reviews on Cancer $2004165423-37$

Received 22 April 2005

Accepted 26 July 2005 